\title{
Condiciones de salud de la población vecina a una planta recicladora de residuos en México' ${ }^{1}$
}

\author{
Horacio Tovalín Ahumada²
}

RESUMEN El presente estudio se realizó en México en una población vecina a una planta recicladora de cinc y de otros polvos metálicos ante la posibilidad de que estas sustancias entraran en contacto con la población y produjeran diferentes efectos nocivos sobre la salud. La planta se sitúa en un municipio cercano a Monterrey, que es la tercera ciudad más poblada de México y la segunda más industrializada.

Se aplicó un diseño transversal comparativo para llevar a cabo la investigación, que duró de septiembre a noviembre de 1994. Mediante un muestreo por cuotas se seleccionaron viviendas en dos zonas —una zona expuesta por vecindad a la planta y otra no expuesta que sirvió de control - hasta lograr el tamaño muestral deseado (621 individuos, a un promedio de cinco por vivienda). En cada domicilio un estudiante de psicología adiestrado aplicó un cuestionario a un familiar mayor de edad para recoger datos sobre la presencia de afecciones agudas y crónicas, trastornos del embarazo y enfermedades congénitas en los integrantes del hogar.

En la población expuesta se aplicaron 127 cuestionarios, que arrojaron información sobre 596 personas, y en la población no expuesta se administraron 147, que proporcionaron datos sobre 743 personas. Las distribuciones por sexo y edad fueron semejantes en las dos poblaciones y el tiempo de residencia en la zona fue ligeramente mayor en los individuos no expuestos.

Los problemas de salud más frecuentes en la población expuesta fueron irritación ocular y de vías respiratorias superiores, alergias, trastornos del sueño, bronquitis, cansancio excesivo, problemas cutáneos, otitis y anemias. Las razones de prevalencias fueron significativas en el caso de erupciones y otras enfermedades cutáneas, cansancio excesivo, trastornos del sueño, infecciones respiratorias superiores, otitis, bronquitis y alergias. Cuando se compararon los dos grupos de mujeres no se encontraron diferencias significativas en las prevalencias de problemas obstétricos, abortos espontáneos o hijos con malformaciones congénitas o bajo peso al nacer.

La sensibilidad del cuestionario utilizado para detectar problemas de salud es pobre y podría haber causado una subestimación de algunas afecciones. No obstante, diferentes sesgos de información fueron controlados y ello permite concluir que la población expuesta tiene un mayor riesgo de padecer ciertas enfermedades y trastornos que la población no expuesta.

1 Próximamente se publicará en esta revista una versión en inglés de este artículo.

2 Universidad Nacional Autónoma de México, Facultad de Estudios Superiores-Zaragoza, México. Las solicitudes de separatas deben dirigirse a este autor a la siguiente dirección postal o electrónica: J. M. Parras, No. 271, Edificio 312 A-402, Colonia Juan Escutia, México DF, C. P. 09110, México. Correo electrónico: tovalin@servidor.unam.mx
Hasta la fecha se han realizado muy pocos estudios destinados a identificar los efectos que pueden ejercer sobre la salud de poblaciones expuestas numerosos residuos y emisiones de plantas donde estos se reciclan, tratan, almacenan o utilizan como materias primas. Las condiciones en que se produce la exposición en estos casos dificultan la realización de este tipo de estudios. A diferencia de las exposiciones laborales, en las cuales los trabajadores se exponen a niveles elevados de sustancias tóxicas, las concentraciones que estas alcanzan en las exposiciones ambientales suelen ser bajas (1).

No obstante, los datos disponibles en la actualidad indican que la exposi- 
ción accidental a algunas sustancias tóxicas depositadas en vertederos industriales puede ser nociva para la salud de las personas que viven cerca de los lugares donde dichas sustancias se utilizan como materia prima. Los efectos tóxicos observados son de diferente tipo y gravedad. Entre ellos destacan malestar general, trastornos respiratorios, cáncer y otras enfermedades de la piel $(2,3)$, leucemias $(4,5)$, malformaciones congénitas (6), bajo peso al nacer (7), alteraciones hepáticas, hipertensión arterial, hernias, trastornos del humor y del carácter y mutaciones genéticas (8).

Si la supervisión a la que están sujetas en México las industrias que procesan estas sustancias no es más rigurosa que la de otros países, es legítimo suponer que en este país también se observen las enfermedades mencionadas en personas expuestas. Por otra parte, cabe esperar que con la aprobación del Tratado de Libre Comercio aumente la entrada en el país de dichas sustancias como materias primas para diversos procesos productivos o para su incineración. En ello estriba la importancia de establecer sistemas de vigilancia destinados, en última instancia, a prevenir cualquier efecto nocivo en la salud de la población y en el medio ambiente (9).

Frente a los hechos señalados, se decidió realizar el presente estudio en la población vecina a una planta recicladora de cinc y otros polvos metálicos retenidos en los dispositivos anticontaminantes de plantas que producen acero a partir de chatarra, y de otros residuos industriales. Los vecinos de dicha planta se habían quejado ante las autoridades por las molestias que les causaban las emisiones de la planta.

Las sustancias tóxicas asociadas con los procesos de recuperación de los polvos de acería son, además del cinc, el arsénico, plomo, cadmio, cobre, cromo y níquel $(10,11)$, así como diversos compuestos orgánicos, que varían según la materia prima utilizada. Varias de esas sustancias son agentes carcinógenos reconocidos. Por ejemplo, el arsénico se asocia con el cáncer de piel y pulmón, según la vía de exposición. El cromo también se asocia con el cáncer de pulmón, trastornos alérgicos, y perforación de mucosas y del tabique nasal. El níquel se ha asociado con cáncer de los senos paranasales y del pulmón, asma y alergias. Por último, el cadmio se ha asociado con cáncer de pulmón, bronquitis, enfisema, nefropatías, enfermedades de la próstata, artritis, menopausia precoz y, cuando la exposición es muy notable, con deformidades óseas (osteomalacia) (12-15).

Otra sustancia tóxica contenida en los residuos mencionados es el plomo, cuyo principal efecto nocivo en la población expuesta por el ambiente se circunscribe al sistema nervioso: en los niños causa alteraciones neuropsicológicas y en los adultos, trastornos hematológicos, cardiovasulares y renales (16). La fuente más importante de exposición ambiental al plomo es la gasolina con tetraetilo de plomo. Se ha señalado, además, que la tierra contaminada con este metal como consecuencia de diversos procesos industriales es una fuente permanente de reexposición $(17,18)$.

Al estudiar los efectos de estas sustancias nocivas es preciso tener en cuenta que las emisiones industriales presentes en el aire, en el agua y en la lluvia constituyen lo que podría denominarse un "caldo de tóxicos" cuyos efectos combinados sobre la salud se desconocen por el momento.

Estas sustancias tóxicas pueden entrar en contacto con la población cuando los vientos y las lluvias arrastran los humos y polvos que los contienen y los introducen en las viviendas. Ahí pueden permanecer durante períodos prolongados y contaminar alimentos y bebidas. Si se depositan en suelos permeables, dichas sustancias pueden contaminar los mantos de agua subterráneos cercanos. El transporte de los contaminantes por diferentes medios permite que ingresen en el organismo por las vías respiratoria, digestiva y, en algunos casos, cutánea, sobre todo en los niños. Si se produce exposición materna, pueden atravesar asimismo la placenta y afectar al feto (19). Estas diversas fuentes y vías de exposición pueden detectarse en cualquier zona industrial donde las plantas carezcan de sistemas anticontaminantes eficientes y donde haya residuos acumulados en los patios $(20,21)$.

Los efectos sobre la salud asociados con la exposición a sustancias tóxicas ambientales se expresan, por una parte, como diferentes molestias y padecimientos agudos $\mathrm{y}$, por otra, como distintas manifestaciones inespecíficas de carácter crónico. Por ello se consideró conveniente conocer la frecuencia con que estas últimas se presentan en la población del estado de Nuevo León, México, donde se encuentra la zona en que se realizó el presente estudio.

En 1994, las principales causas de mortalidad en ese estado fueron las enfermedades del corazón, los tumores, los accidentes y la diabetes mellitus. Los tumores más frecuentes notificados ese mismo año fueron las leucemias y los cánceres pulmonares, gástricos y mamarios. Las tasas de mortalidad por cáncer de pulmón y mama y por leucemias en Nuevo León fueron más elevadas que las nacionales (cuadro 1). Estos datos sugieren la presencia en la población de la zona de enfermedades que pueden asociarse con contaminantes ambientales y por ello es necesario analizar si son atribuibles a la exposición a las sustancias tóxicas emitidas por las industrias locales.

CUADRO 1. Tasas de mortalidad ( $\times 100000$ habitantes) por diversas causas en el estado de Nuevo León y en todo México, 1986

\begin{tabular}{lcr}
\hline & \multicolumn{2}{c}{ Tasa de mortalidad } \\
\cline { 2 - 3 } Causa de muerte & Nuevo León & México \\
\hline Aparato circulatorio & 87,4 & 67,5 \\
Tumores malignos & & \\
$\quad$ (total) & 61,1 & 51,6 \\
$\quad$ Traquea, bronquio, & & \\
$\quad$ pulmón & 10,7 & 6,5 \\
Mama & 4,8 & 3,1 \\
Estómago & 4,2 & 5,2 \\
$\quad$ Leucemias & 3,1 & 3,0 \\
Accidentes & 43,8 & 41,4 \\
Diabetes mellitus & 32,3 & 33,7 \\
Enfermedad & & \\
$\quad$ cardiovascular & 28,0 & 25,2 \\
Malformaciones & & \\
$\quad$ congénitas & 9,9 & 10,3 \\
Bronquitis & 4,5 & 9,2 \\
Asma & 1,4 & 2,6 \\
\hline
\end{tabular}

Fuente: Referencia 27. 


\section{MATERIALES Y MÉTODOS}

La población del estudio se compuso de los habitantes vecinos de una planta recicladora de residuos peligrosos procedentes de una acería, que se encuentra en un municipio cercano a la ciudad de Monterrey, la tercera ciudad más poblada de México y la segunda más industrializada.

Para realizar el estudio se empleó un diseño transversal. Al iniciarlo, se definieron dos grupos - uno de expuestos y otro de no expuestos a las fuentes de contaminación (22) - cuyo estado de salud se evaluó una sola vez entre septiembre y noviembre de 1994. Las personas que vivían en la zona potencialmente expuesta eran de un nivel socioeconómico medio (muchos responsables de familia eran profesionales) y disponían de todo tipo de servicios y de viviendas de buena calidad o de lujo. Se excluyó de esta investigación a los integrantes de una colonia marginal cercana a la planta por no contarse con un grupo de comparación adecuado. En la zona estudiada no existía ningún otro establecimiento industrial y había poco tráfico en las calles. Para fines de comparación (población no expuesta) se seleccionaron personas que residían en colonias similares en sus características a las de los individuos expuestos, salvo que se localizaban al sur de Monterrey, fuera de las zonas industriales.

Para calcular el tamaño muestral necesario se utilizó la fórmula de Fleiss (23). Se eligieron los siguientes parámetros: grado de significación estadística, 0,05; potencia estadística, 0,9 ; probabilidad de presentar diferentes signos y síntomas asociados con la exposición a las sustancias tóxicas mencionadas, 0,10 en los expuestos y 0,05 en los no expuestos. Con estos datos, el tamaño muestral necesario se calculó en 621 individuos.

La unidad del estudio estuvo constituida por cada uno de los domicilios ubicados en las zonas donde se administró un cuestionario familiar. Como se estimó que en cada hogar vivían cinco personas en promedio, al dividir los 621 integrantes de la muestra por cinco se obtuvo un total de 124 cues- tionarios familiares. Esta cifra se aumentó a 130 en previsión de ausencias y faltas de respuesta. Como hogar se consideró el conjunto de personas que dependen de un solo ingreso económico y que viven bajo un mismo techo, de manera que puede haber más de un hogar por domicilio si los integrantes dependen de más de un ingreso.

En ausencia de un marco muestral de los hogares en el área estudiada, se realizó un muestreo según cuotas que debían cubrirse en cada zona, procurando abarcar todas las viviendas situadas en las cercanías de la planta. El número total de cuestionarios programados para el área expuesta fue de 130 y para la no expuesta, de 150.

La información sobre las condiciones de salud se recabó por medio de un cuestionario familiar administrado en cada domicilio a un informante calificado, entendiéndose por tal a toda persona mayor de edad y responsable que viviera en el hogar y pudiera aportar la información precisada. El cuestionario se dirigió a identificar a todos los integrantes del hogar, a determinar el tiempo que habían vivido en la zona, y a investigar en cualquier miembro la presencia de síntomas y signos inespecíficos agudos, enfermedades crónicas como el cáncer, trastornos del embarazo (amenaza de aborto, aborto espontáneo, parto prematuro y bajo peso al nacer) y enfermedades o malformaciones congénitas. Con el fin de lograr mayor exactitud en su registro, se determinó si había signos y síntomas inespecíficos en el momento en que se hizo la encuesta. En cambio, en el caso de enfermedades crónicas, se investigó su presencia durante todo el período de residencia en la zona estudiada. Al finalizar la encuesta se preguntó al informante calificado si asociaba la contaminación producida por alguna de las plantas cercanas con los problemas de salud de su familia y, en caso afirmativo, a qué planta podrían atribuirse.

El cuestionario fue administrado por estudiantes de psicología debidamente capacitados. Con el propósito de evitar cualquier sesgo debido al observador (24), al capacitar a estos encuestadores se les ocultó el verdadero objetivo del estudio -identificar los efectos sobre la salud atribuibles a las emisiones de la recicladora- y se les indicó, más bien, que su propósito era conocer los efectos de la contaminación ambiental en general en varias colonias de la zona metropolitana de Monterrey. De este modo se aseguró que no hubiera diferencias en la manera de entrevistar a los individuos expuestos y no expuestos.

La información obtenida se procesó con el programa dBase III-plus y los análisis estadísticos se llevaron a cabo con el programa Epi Info 5.1. Para analizar los datos se calcularon las distribuciones de frecuencias de las principales molestias y enfermedades, aplicándose pruebas de hipótesis para diferencias entre proporciones. Para comparar las posibilidades de expuestos y no expuestos de presentar los distintos trastornos considerados se calcularon las razones de prevalencias de dichos trastornos, junto con sus intervalos de confianza de $95 \%$.

\section{RESULTADOS}

En el grupo de expuestos se administraron en total 127 cuestionarios y se obtuvo información de 596 personas; en el de no expuestos se administraron 147 cuestionarios y se recabó información de 743 personas. Por tanto, se cubrieron prácticamente todas las viviendas a las que en principio se debían realizar entrevistas. Ningún entrevistado se opuso a dar información. La única causa de falta de respuesta fue el no haber encontrado personas en la vivienda.

De la población expuesta, $50,7 \%$ se compuso de mujeres y 49,3\%, de hombres. En el grupo de no expuestos estos porcentajes fueron, respectivamente, 49,7 y 51,3\%. La distribución de edades de ambos grupos fue similar y no se observaron diferencias estadísticamente significativas entre las proporciones de personas en cada grupo de edad. El tiempo de residencia medio de los individuos expuestos fue de 8,49 años y el de los no expuestos, de 11,0 años.

De los informantes calificados del grupo de expuestos, $66,7 \%$ declararon 
que sus molestias estaban relacionadas con los contaminantes emitidos por alguna planta cercana y $48 \%$ identificaron la planta de la vecindad como la fuente responsable. De los informantes del grupo de no expuestos, $12,9 \%$ asociaron las molestias con las emisiones de una planta cercana y solo $4(2,8 \%)$ señalaron una planta en concreto.

Los problemas de salud más frecuentes en los expuestos fueron irritación de los ojos y de las vías respiratorias superiores, alergias, trastornos del sueño, bronquitis, sensación de fatiga excesiva, problemas de la piel, infecciones del oído y anemias. En los no expuestos se observaron los mismos trastornos, aunque la frecuencia de algunos de ellos fue más baja (cuadro 2 ). Las proporciones de fumadores en ambos grupos fueron similares $(10,4 \%$ en los individuos expuestos y $11,2 \%$ en los no expuestos).

Las razones de prevalencias de erupciones y otras enfermedades de la piel, cansancio excesivo, trastornos del sueño, infecciones de las vías respiratorias superiores, bronquitis, otitis y alergias fueron mayores en los expuestos que en los no expuestos (véase el cuadro 2). ${ }^{3}$

Con objeto de controlar posibles factores de confusión al estimar las razones de prevalencias, las prevalencias se ajustaron según el tiempo de residencia en el área. Estas estimaciones no pueden considerarse distintas de las no ajustadas. Además, las prevalencias de irritación de los ojos y de infecciones de las vías respiratorias superiores se ajustaron según el tiempo de residencia en el área y el hábito tabáquico, y el valor estimado (1,8; IC95\%: 1,3 - 2,4) fue muy similar al no ajustado. Tampoco se observaron diferencias entre las prevalencias brutas de los distintos trastornos y las prevalencias ajustadas según la edad.

\footnotetext{
Como puede observarse, en este cuadro también se han incluido razones de prevalencias cuyos intervalos de confianza contienen el valor 1,0. Es posible que la prevalencia de algunos trastornos en los dos grupos se haya subestimado por la baja sensibilidad del instrumento medidor empleado. La anemia se ha omitido por no haberse encontrado ningún caso en individuos no expuestos.
}

CUADRO 2. Prevalencias y razones de prevalencias de distintos trastornos y enfermedades considerados, en expuestos y no expuestos del área del estudio. Nuevo León, México, 1994

\begin{tabular}{|c|c|c|c|c|c|c|}
\hline \multirow[b]{2}{*}{ Trastorno } & \multicolumn{2}{|c|}{ Expuestos } & \multicolumn{2}{|c|}{ No expuestos } & \multirow{2}{*}{\multicolumn{2}{|c|}{$\begin{array}{c}\text { Razón de } \\
\text { prevalencias (IC95\%) }\end{array}$}} \\
\hline & No. & $\%$ & No. & $\%$ & & \\
\hline \multicolumn{7}{|l|}{ Irritación de ojos y vías } \\
\hline respiratorias superiores & 103 & 17,3 & 66 & 8,8 & 1,9 & $(1,5-2,6)$ \\
\hline Alergias & 92 & 15,4 & 82 & 11,0 & 1,5 & $(1,1-2,0)$ \\
\hline Alteraciones del sueño & 52 & 8,7 & 28 & 3,7 & 2,3 & $(1,5-4,0)$ \\
\hline Bronquitis & 49 & 8,2 & 34 & 4,6 & 1,8 & $(1,2-2,7)$ \\
\hline Cansancio excesivo & 42 & 7,0 & 19 & 2,5 & 2,8 & $(1,6-4,7)$ \\
\hline Problemas cutáneos & 36 & 6,0 & 20 & 2,7 & 2,2 & $(1,3-3,8)$ \\
\hline Erupciones & 36 & 6,0 & 16 & 2,2 & 2,8 & $(1,6-5,0)$ \\
\hline Otitis & 44 & 7,4 & 18 & 2,4 & 2,5 & $(1,4-4,3)$ \\
\hline Anemia & 8 & 1,3 & 0 & 0 & - & - \\
\hline Asma & 23 & 3,8 & 24 & 3,2 & 1,2 & $(0,7-2,1)$ \\
\hline Nefropatías & 21 & 3,5 & 23 & 3,0 & 1,1 & $(0,6-2,0)$ \\
\hline Hernias & 16 & 2,6 & 15 & 2,0 & 1,3 & $(0,6-2,7)$ \\
\hline Hepatopatías & 4 & 0,6 & 3 & 0,4 & 1,6 & $(0,3-9,4)$ \\
\hline
\end{tabular}

Las razones de prevalencias de los trastornos estudiados aparecen en el cuadro 3 desglosadas por sexo. En las mujeres expuestas, las prevalencias de trastornos cutáneos, otitis, cansancio excesivo, trastornos del sueño y alergias fueron más altas que en las no expuestas y en los hombres se observaron resultados similares, si bien las razones de prevalencias estimadas para la mayor parte de los problemas fueron más altas en los hombres.

Como ilustra el cuadro 4 , los datos no permiten afirmar que las prevalencias de problemas obstétricos, malformaciones congénitas, abortos y bajo peso al nacer en las mujeres expuestas fuesen distintas de las encontradas en las no expuestas.

CUADRO 3. Razones de prevalencias, desglosadas por sexo, de distintos trastornos y enfermedades en expuestos y no expuestos del área del estudio. Nuevo León, México, 1994

\begin{tabular}{lrr}
\hline & \multicolumn{1}{c}{ Razón de prevalencias (IC95\%) } \\
\cline { 2 - 3 } \multicolumn{1}{c}{ Trastorno } & Mujeres & Hombres \\
\hline Problemas cutáneos & $3,76(1,63-8,68)$ & $1,35(0,98-3,12)$ \\
Otitis & $2,72(1,34-5,53)$ & $5,99(2,31-15,57)$ \\
Erupciones cutáneas & $2,13(0,95-4,75)$ & $3,52(1,36-9,42)$ \\
Trastornos del sueño & $2,58(1,36-4,89)$ & $2,61(1,33-5,12)$ \\
Alergias & $1,45(1,06-2,12)$ & $1,68(1,14-2,50)$ \\
Cansancio excesivo & $2,49(1,28-4,88)$ & $3,27(1,28-8,31)$ \\
Irritación de ojos y vías respiratorias superiores & $1,8(1,18-2,73)$ & $2,08(1,41-3,1)$ \\
\hline
\end{tabular}


CUADRO 4. Prevalencias y razones de prevalencias de trastornos reproductivos en las mujeres expuestas y no expuestas del área del estudio. Nuevo León, México, 1994

\begin{tabular}{|c|c|c|c|c|c|c|}
\hline \multirow{3}{*}{$\begin{array}{l}\text { Trastorno reproductivo } \\
\text { Aborto espontáneo }\end{array}$} & \multicolumn{2}{|c|}{ Expuestas } & \multicolumn{2}{|c|}{ No expuestas } & \multirow{2}{*}{\multicolumn{2}{|c|}{$\begin{array}{c}\text { Razón de } \\
\text { prevalencias (IC95\%) }\end{array}$}} \\
\hline & No. & $\%$ & No. & $\%$ & & \\
\hline & 13 & 11,2 & 8 & 5,6 & 1,9 & $(0,8-4,6)$ \\
\hline Amenaza de aborto & 8 & 6,8 & 6 & 4,2 & 1,6 & $(0,6-4,5)$ \\
\hline Malformaciones congénitas & 4 & 3,4 & 2 & 1,4 & 2,4 & $(0,5-13,1)$ \\
\hline Parto prematuro & 3 & 2,5 & 10 & 7,0 & 0,4 & $(0,1-1,3)$ \\
\hline Bajo peso al nacer & 2 & 1,7 & 2 & 1,4 & 1,2 & $(0,2-8,5)$ \\
\hline
\end{tabular}

a suponer que las exposiciones de todos los entrevistados habían sido similares. La confusión producida por la exposición a contaminantes atmosféricos se controla comparando dos poblaciones sometidas a grados semejantes de contaminación ambiental general, pero con distinta exposición a tóxicos industriales. Por otra parte, el porcentaje relativamente alto de los informantes que relacionaron la contaminación producida por las plantas con el estado de salud de su familia es un dato adicional que apoya la posible presencia de una relación.

Puede ser que en el momento de obtener la información con el instrumento utilizado para tal fin se hayan introducido otros sesgos. En este estudio puede descartarse un sesgo debido al observador, ya que los entrevistadores no conocían el objetivo del estudio. Además, por la forma como se elaboró el cuestionario se evitó suscitar en los entrevistados la idea de que sus problemas de salud estuviesen relacionados con la contaminación procedente de las fábricas.

La sensibilidad del cuestionario para detectar problemas de salud es pobre y, por ello, es posible que algu- nos problemas de salud hayan sido subestimados por los entrevistados. También es posible que a ello se sume el efecto de un sesgo de olvido o desconocimiento por parte del informante. No obstante, es probable que este sesgo haya sido similar en los expuestos y no expuestos y en ese caso sus efectos se compensan. Tampoco puede descartarse la posibilidad de que se hayan subestimado los riesgos de algunas enfermedades (26).

La similar frecuencia de enfermedades crónicas detectada en los dos grupos estudiados puede deberse, como sugiere el patrón de mortalidad del estado de Nuevo León, a la elevada frecuencia de algunas enfermedades crónicas en la población en general (27). En otras palabras, cualquier efecto tóxico producido por las exposiciones consideradas en este estudio podría quedar oculto en el conjunto de las numerosas enfermedades crónicas producidas por otras causas. En futuros estudios debería explorarse la existencia de esta asociación.

El no haber encontrado diferencias entre las razones de prevalencias brutas y las ajustadas según el tiempo de residencia en el área estudiada se ex- plica probablemente por el tipo de enfermedades y trastornos notificados, que en su mayor parte fueron de tipo agudo o subagudo. Lo mismo puede afirmarse en cuanto a los efectos de la edad, el tiempo de residencia y el tabaquismo. El menor tiempo de residencia en la zona reduce las posibilidades de encontrar ciertas enfermedades asociadas con la exposición a los tóxicos emitidos, como los tumores de desarrollo lento. Asimismo, es preciso tener en cuenta la dificultad que entraña la obtención de información confiable sobre los problemas reproductivos, cuya frecuencia no cabe descartar que se haya subestimado.

Por otra parte, es posible que se hayan perdido personas cuyos trastornos crónicos fueron producidos o agravados por las sustancias tóxicas emitidas en la zona. Algunas podrían haber abandonado la zona para aliviarse de enfermedades respiratorias producidas por ellas. Ello supone la pérdida de casos expuestos $\mathrm{y}$, por ende, una asociación subestimada.

Los resultados de este estudio sugieren que las personas expuestas tienen una mayor posibilidad de padecer las enfermedades y trastornos considerados que las personas no expuestas.

Agradecimiento. Los autores agradecen a Fernando Bejarano, responsable de la Campaña de la Oficina de Tóxicos de Greenpeace-México, a Raúl Rubio Cano, a Juan de Dios Sánchez Martínez y a Federico Lazcano Ramírez su iniciativa y el apoyo que brindaron a este estudio, así como la ayuda prestada por todos aquellos que contribuyeron a obtener la información necesaria para realizarlo.

\section{REFERENCIAS}

1. National Research Council. Environmental epidemiology. Washington, DC: National Academy Press; 1991.

2. British Medical Association. Hazardous waste and human health. Oxford: Oxford University Press; 1990.
3. Hertzman CM. Upper Ottawa landfill site health study. Environ Health Perspect 1987;75: 173-195.

4. Neuberger JS, Mulhall M, Pomatto MC, Sheverbush J, Hassanein RS. Health problems in Galena, Kansas (USA): a heavy metal min- ing superfund site. Sci Total Environ 1990;94: 261-272.

5. Ozonoff DM, Colten ME, Cupples A, Heeren $\mathrm{T}$, Schatzkin A, Mangione $\mathrm{T}$, et al. Health problems reported by residents of a neighborhood contaminated by a haz- 
ardous waste facility. Am J Ind Med 1987; 11:581-597.

6. Lagakos SW, Wenssen BJ, Zelen M. An analysis of contaminated well water and health effects in Woburn, Massachusetts. J Am Stat Assoc 1986;81:583-596.

7. Goldberg SJ, Lebowitz MD, Graver EJ, Hicks $\mathrm{S}$. An association of human congenital cardiac malformations and drinking water contaminants. J Am College Cardiol 1990;16:155-164.

8. Harris R, Rodricks J, Clarck S, Papadopoulos S. Adverse health effects at a Tennessee hazardous waste disposal site. En: Andelman J, Underhill D. Health effects from hazardous waste sites. Chelsea, Michigan: Lewis Publishers; 1990:221-240.

9. Stebbins KR. Garbage imperialism: health implications of dumping hazardous waste in Third World countries. Med Anthropol 1992; 15:81-102.

10. Manahan S. Hazardous waste chemistry, toxicology and treatment. Chelsea, Michigan: Lewis Publishers; 1990.

11. Cralley LV, Cralley LJ. Vol I, Process flows. En: Industrial hygiene aspects of plant operation. New York: McMillan Publishing; 1982.

12. Heith L, Walters D. Vol. III. Standards and regulations En: The National Toxicology Program. Chelsea, Michigan: Lewis Publishers; 1992.
13. Lewis R. Hazardous chemicals: desk reference. New York: Van Nostrand Reinhold; 1993.

14. Lippman M. Environmental toxicants. New York: Van Nostrand Reinhold; 1991.

15. Landrigan P. Occupational and community exposures to toxic metals: lead, cadmium, mercury and arsenic. Western J Med 1982; 11:531-539.

16. Gossed T, Briker D. Principles of clinical toxicology. New York: Raven Press; 1990:162-193.

17. Academia Americana de Pediatría. Intoxicación por plomo: de la detección a la prevención primaria. Salud Publica Mex 1995;37: 264-275.

18. Agency for Toxic Substances and Disease Registry. Toxicological profile for lead. Atlanta: U.S. Department of Health and Human Services; 1993.

19. Agency for Toxic Substances and Disease Registry. Public Health assessment: guidance manual. Chelsea, Michigan: Lewis Publishers; 1992.

20. Agency for Toxic Substances and Disease Registry. The Crystal Chemical Company: arsenic exposure study. Houston: ATSDR, Epidemiology and Medicine Branch; 1989.

21. LaGoy P, Nisbet I, Schultz C. The endangerment assessment for the smuggler mountain site, Pitkin County, Colorado. En: Pausten- bach $\mathrm{D}$. The risk assessment of environmental and human health hazards. New York: John Wiley and Sons; 1989:505-525.

22. Kleinbaum DG, Kupper LL, Morgensten H. Epidemiologic research. New York: Van Nostrand Reinhold; 1982.

23. Fleiss J. Statistical methods for rates and proportions. New York: John Wiley and Sons; 1985.

24. Andersson N. Distractores y modificadores de efecto: conceptos claves en la epidemiología moderna. Prioridades Salud 1991;2: 52-56.

25. Andersson N. Los problemas con el muestreo probabilístico en la selección de los sitios centinela. Prioridades Salud 1991;2:30-35.

26. Kaye WE, Hall HI, Lybarger JA. Recall bias in disease status with perceived exposure to hazardous substances. Ann Epidemiol 1994;4: 393-397.

27. Instituto Nacional de Estadística, Geografía e Informática. Tabulaciones por defunciones. México, DF: INEGI; 1990.

Manuscrito recibido el 3 de abril de 1996 y aprobado para publicación en versión revisada el 27 de enero de 1997.

ABSTRACT This study was conducted in Mexico among residents of an area near a recycling plant for zinc and other metallic dusts to find out if these substances produce various adverse health effects in the population that may come in contact with them. The

Health conditions in a population living near a waste recycling plant in Mexico plant is in a municipality close to Monterrey, which is Mexico's third most populous and second most industrialized city.

A cross-sectional comparative design was used for the research, which took place from September to November 1994. By means of quota sampling, houses were selected in two areas-one exposed, by virtue of its proximity to the plant, and the other unexposed, which served as a control-until the desired sample size was reached (621 individuals, an average of five per household). In each dwelling, a trained psychology student administered a questionnaire to one of the older family members to gather data on the presence of acute and chronic illnesses, problems during pregnancy, and congenital illnesses among the household members.

In the exposed population, 127 questionnaires were completed, providing information on 596 persons; in the unexposed population, data on 743 persons were gathered via 147 questionnaires. The sex and age distributions were similar in the two populations, and the length of residence in their respective area was slightly longer among unexposed individuals.

The most frequently reported health problems in the exposed population were irritation of the eyes and upper respiratory tract, allergies, sleep disturbances, bronchitis, fatigue, skin problems, ear infections, and anemia. The prevalence ratios for the exposed versus unexposed populations were significant with regard to skin eruptions and other skin diseases, fatigue, sleep disturbances, upper respiratory infections, ear infections, bronchitis, and allergies. Women from the two groups did not show significant differences in the prevalence of obstetric problems, miscarriages, or children born with congenital deformities or low birthweight.

The sensitivity of the questionnaire used to detect health problems was poor, which could have resulted in an underestimation of some disorders. Nevertheless, various types of information bias were controlled, permitting the conclusion that the exposed population had a greater risk of suffering certain diseases and disorders than the unexposed population. 